\title{
The void abundance with non-gaussian primordial perturbations
}

\author{
Marc Kamionkowski, ${ }^{a}{\text { Licia } \text { Verde }^{b} \text { and Raul Jimenez }}^{b}$ \\ ${ }^{a}$ California Institute of Technology, \\ Mail Code 130-33, Pasadena, CA 91125, U.S.A. \\ ${ }^{b}$ ICREA \& Institute of Space Sciences (CSIC-IEEC), \\ Campus UAB, Bellaterra, Spain \\ E-mail: kamion@tapir.caltech.edu, lverde@astro.princeton.edu, raul@ieec.uab.es
}

Received September 2, 2008

Revised November 21, 2008

Accepted November 29, 2008

Published January 8, 2009

\begin{abstract}
We use a Press-Schechter-like calculation to study how the abundance of voids changes in models with non-Gaussian initial conditions. While a positive skewness increases the cluster abundance, a negative skewness does the same for the void abundance. We determine the dependence of the void abundance on the non-Gaussianity parameter $f_{\text {nl }}$ for the local-model bispectrum - which approximates the bispectrum in some multi-field inflation models - and for the equilateral bispectrum, which approximates the bispectrum in singlefield slow-roll inflation and in string-inspired DBI models of inflation. We show that the void abundance in large-scale-structure surveys currently being considered should probe values as small as $f_{\mathrm{nl}} \lesssim 10$ and $f_{\mathrm{nl}}^{\mathrm{eq}} \lesssim 30$, over distance scales $\sim 10 \mathrm{Mpc}$.
\end{abstract}

Keywords: inflation, physics of the early universe, superclusters and voids, power spectrum 


\section{Contents}

1 Introduction $\quad 1$

2 The Press-Schechter abundance $\quad 2$

2.1 Void abundance: Gaussian initial conditions 3

2.2 Void abundance with non-Gaussian initial conditions 3

3 The skewness 4

4 Results 5

$\begin{array}{lll}5 & \text { Discussion } & 7\end{array}$

\section{Introduction}

The paradigm of cosmological structure formation from a spectrum of primordial perturbations like those predicted by inflation has now been fairly well established by cosmic microwave background (CMB) experiments [1]. We are thus now motivated to test more precisely the predictions of inflation and to look for possible deviations. One of several such possibilities is measurement of departures from Gaussianity of the initial perturbations (see, e.g., ref. [2] and references therein). The simplest slow-roll single-field models of inflation predict that primordial perturbations should be very closely Gaussian [3], but with predictably small departures from Gaussianity $[4,5]$. Multi-field [6] models, such as the curvaton model [7], and string-inspired DBI [8] inflationary models can produce larger deviations from non-Gaussianity.

Departures from primordial Gaussianity can be sought in the CMB [9-11], large-scale structure (LSS) [12], and the abundances and properties of the most massive gravitationallybound objects in the Universe today or at high redshift [13-15]. The CMB provides a more powerful and clean probe of primordial non-Gaussianity than direct measurement of the bispectrum in low-redshift LSS surveys in models with scale-invariant non-Gaussianity [10], although biasing may amplify the effects of non-Gaussianity on LSS to the level where they may be comparable in detectability to the CMB [16-19]. Measurements of the cluster abundance may do better than the CMB and LSS if the non-Gaussianity is not scale-invariant [20], as may occur in DBI models. What is clear, however, is that the thorny systematic effects that enter in all of these approaches will require that a variety of complementary avenues be taken to establish a robust detection of non-Gaussianity.

Voids have been considered as probes of cosmology, but no systematic study has been carried out for voids as probes of primordial non-gaussianity [21]. In this paper, we consider the abundance of voids as a test of the distribution of the primordial perturbations. Galaxy clusters form at the highest overdensities of the primordial density field and thus probe the high-density tail of the primordial density distribution function. Similarly, voids form in low-density regions and should thus probe the low-density tail of the distribution function. If there is a large negative skewness, the void-size distribution function will be increased at 
the largest void sizes and decreased at smaller void sizes, opposite to the effect on the cluster mass function.

In section 2, we develop a Press-Schechter (PS) estimate of the void abundance for Gaussian initial conditions. This PS-like calculation is far from state of the art $[22,23]$ for Gaussian initial conditions. However, it is easily generalized to non-Gaussian initial conditions and should be sufficiently reliable to estimate the fractional effects of non-Gaussianity on the void abundance (as it describes well the halo abundance [24]. In section 3, we discuss the relation between the skewness and the non-Gaussian parameter $f_{\mathrm{nl}}$ for the local model [4], which approximates the non-Gaussianity in multi-field models, and the equilateral model [5], which approximates that in single-field slow-roll inflation and in string-inspired DBI models. In section 4, we provide results of the void-abundance calculation, and we estimate the smallest $f_{\mathrm{nl}}$, for the local model and for the equilateral model (with and without scale dependence) that should be detectable in several surveys currently under study. In section 5 , we make some concluding remarks and outline further steps that must be taken before the void abundance can be used to probe non-Gaussianity.

\section{The Press-Schechter abundance}

We begin by developing a PS-like calculation of the void abundance, but we first review the standard Press-Schechter calculation of the halo mass function.

Formation of a bound halo requires a linear-theory density fluctuation $\delta_{R}>\delta_{c} \simeq 1.69$, smoothed on scale $R .{ }^{1}$ The smoothing radius $R$ defines the halo mass $M$ via $M=(4 \pi / 3) \rho_{b} R^{3}$, where $\rho_{b}$ is the mean nonrelativistic-matter density.

The differential abundance of dark-matter halos as a function of mass and redshift is

$$
\frac{d n}{d M}=f \frac{\rho_{b}}{M}\left|\frac{d P\left(>\delta_{c} \mid z, M\right)}{d M}\right|
$$

where $P\left(>\delta_{c} \mid z, M\right)$ denotes the probability that $\delta_{R}$ lie above the threshold for collapse $\delta_{c}$. For Gaussian initial conditions,

$$
P\left(>\delta_{c} \mid z, R\right)=\frac{1}{\sqrt{2 \pi}} \frac{\sigma_{R}}{\delta_{c}} \exp \left[-\frac{1}{2} \frac{\delta_{c}^{2}}{\sigma_{R}^{2}}\right]
$$

where $\sigma_{R}$ denotes the rms mass fluctuation on a scale $R$, and there is an implicit redshift dependence in $\sigma_{R}$. We then introduce the Press-Schechter swindle - i.e., that every mass element in an underdense region gets absorbed into the nearest overdensity — by introducing a factor of 2 in the abundance. The differential abundance of halos is then

$$
\frac{d n}{d M} d M=\sqrt{\frac{2}{\pi}} \frac{\rho_{b}}{M^{2}} \frac{\delta_{c}}{\sigma_{M}}\left|\frac{d \ln \sigma_{M}}{d \ln M}\right| e^{-\delta_{c}^{2} / 2 \sigma_{M}^{2}} d M .
$$

The mass function is then normalized so that $\int_{0}^{\infty} M(d n / d M) d M=\rho_{b}$; i.e., every mass element in the Universe is housed somewhere.

\footnotetext{
${ }^{1}$ This value $\delta_{c}=1.69$ is strictly appropriate for an Einstein-de Sitter Universe; for different cosmologies this number varies slightly and has a very mild dependence on redshift.
} 


\subsection{Void abundance: Gaussian initial conditions}

A similar calculation can be used to estimate the void abdundance. In the PS description, every mass element is in a virialized structure (a halo) of some mass, and the densities of these objects are all $\gtrsim 200$ times the mean density. This means that $\gtrsim 99.5 \%$ of the volume of the Universe is empty. A void distribution function can be derived in a way analogous to the PS mass function by realizing that negative density fluctuations grow into voids (as opposed to positive-density fluctuations, which grow into bound objects), and that there is a critical underdensity $\delta_{v}$ for producing a void, to replace $\delta_{c}$, the critical overdensity for producing bound objects.

In principle, $\delta_{v}$ can be calculated from theory. If the voids are spherical, the value of $\delta_{v}$ determines the fractional underdensity in the void, as shown, e.g., in figure 1 of ref. [23]. For example, if we define voids to be regions that have a density half of the mean cosmological density, then $\delta_{v} \simeq-0.7$. If voids are defined to be even more underdense, then $\delta_{v}$ will become even more negative. Realistic values of $\delta_{v}$ are likely to be $\delta_{v} \gtrsim-1$. Beyond this, isolated voids undergo shell crossing, and in a realistic Universe, with many voids, voids are likely to run into each other [25]. Since $\left|\delta_{v}\right|$ is typically smaller than $\delta_{c}$, voids generally probe structure on larger scales than those probed by clusters. Strictly speaking, the precise value of $\delta_{v}$ will depend on the precise definition of a void; it will need to be determined through simulations and mock catalog surveys. For now, we leave it as a phenomenological parameter and show results for a variety of values of $\delta_{v}$. As we will see, our basic conclusions will not depend on its precise value.

The radius $R$ of a spherical volume in which a mass $M$ has been cleared out is $R=$ $\left(3 M / 4 \pi \rho_{b}\right)^{1 / 3}$. We thus derive $(d n / d R)$, the differential abundance of voids of diameter $R$,

$$
\frac{d n}{d R}=\frac{9}{2 \pi^{2}} \sqrt{\frac{\pi}{2}} \frac{1}{R^{4}} \frac{\left|\delta_{v}\right|}{\sigma_{M}}\left|\frac{d \ln \sigma_{M}}{d \ln M}\right| e^{-\delta_{v}^{2} / 2 \sigma_{M}^{2}}
$$

We here introduce again the Press-Schechter swindle, to take into account the fact that each underdense region expands by a factor of two so that the entire (actually, 99.5\% of the) volume of the Universe is occupied by a voids of radii $R$ with the distribution $d n / d R$; i.e., the size distribution is normalized so that

$$
\int_{0}^{\infty} \frac{d n}{d R} \frac{4 \pi R^{3}}{3} d R=1
$$

\subsection{Void abundance with non-Gaussian initial conditions}

The modification of eq. (2.4) when there is a small primordial skewness $S_{3, M}$ (which may most generally depend on the mass scale $M$ ) then follows from the analogous modification for the halo abundance in refs. $[14,15,20]$. The only subtlety is that $\delta_{v}$ is now a negative quantity (since clusters come from overdensities, while voids come from underdensities). In fact $P_{<\delta}=1-P_{>\delta}$ thus $\left|d P_{<\delta_{v}} / d M\right|=\left|d P_{>\delta_{v}} / d M\right|$. We can still use $\left|\delta_{v}\right|$ provided that eq. (2.4) is replaced by (see [20])

$$
\begin{aligned}
\frac{d n}{d R}=\frac{9}{2 \pi^{2}} \sqrt{\frac{\pi}{2}} \frac{1}{R^{4}} e^{-\delta_{v}^{2} / 2 \sigma_{M}^{2}}\{\mid & \left|\frac{d \ln \sigma_{M}}{d \ln M}\right|\left[\frac{\left|\delta_{v}\right|}{\sigma_{M}}-\frac{S_{3} \sigma_{M}}{6}\left(\frac{\delta_{v}^{4}}{\sigma_{M}^{4}}-2 \frac{\delta_{v}^{2}}{\sigma_{M}^{2}}-1\right)\right] \\
& \left.+\frac{1}{6} \frac{d S_{3}}{d M} \sigma_{M}\left(\frac{\delta_{v}^{2}}{\sigma_{M}^{2}}-1\right)\right\}
\end{aligned}
$$




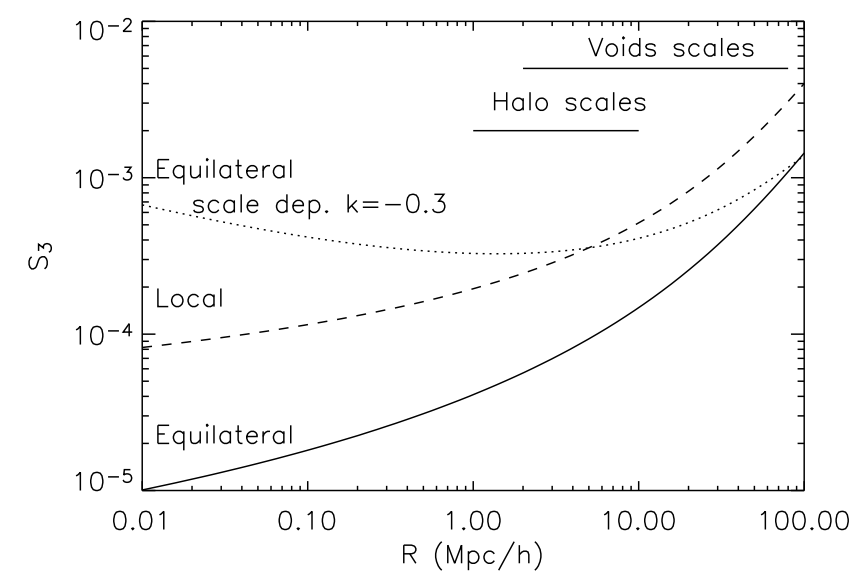

Figure 1: Skewness $S_{3, R}$ for a non-Gaussianity parameter $f_{\mathrm{nl}}=1$ as function of radius $R$. The dashed line is the local non-Gaussian model; the solid line is the equilateral model; and the dotted line is the DBI-type equilateral model for a scale-dependence parameter $\kappa=-0.3$. See ref. [20] for details. We also indicate the scales probed by halo and void abundances. The void range in the figure is only indicative; in detail, the range of scales probed by voids will depend on the value of the underdensity threshold used to define the void.

The derivation of this formula relies heavily on the Press-Schechter approach to the description of voids abundance. Refs. [22, 23] show that in the gaussian case this is a good approximation for large voids; for small voids this approach does not work as well. Thus the quantitative interpretation of these results for small voids should be interpreted with care. We believe however that this approach can still quantify the usefulness of looking at the void abundance. Rigorously quantitative results will need to be calibrated on cosmological simulations and mock survey catalogs.

\section{The skewness}

According to eq. (2.6), the void abundance depends on the non-Gaussianity only through the skewness $S_{3, R}$. The skewness then depends on the detailed form of the bispectrum. There are a variety of bispectra considered in the literature. The standard "working-horse" model for non-Gaussianity is perhaps the local model, which features a bispectrum that arises from multi-field inflation models. There is then the equilateral model, which approximates the bispectrum in DBI models. DBI models can also allow for a scale-dependent non-Gaussianity, with power-law index $\kappa$. See ref. $[5,20]$ for definitions and further details.

In figure 1 , we plot the skewness $S_{3, R}$ for a non-Gaussianity parameter $f_{\mathrm{nl}}=1$, as a function of $R$ for the local model, the equilateral model, and the equilateral model with a scale-dependence parameter $\kappa=-0.3$. The skewness in the equilateral model is about a factor 3 smaller than that for the local model. We thus infer that constraints to $f_{\mathrm{nl}}$ for the local model that derive simply from $S_{3, R}$ will be a factor 3 more stringent than for the equilateral model. An equilateral model with scale dependence characterized by $\kappa=-0.3$ will have constraints similar to those for the local model. 


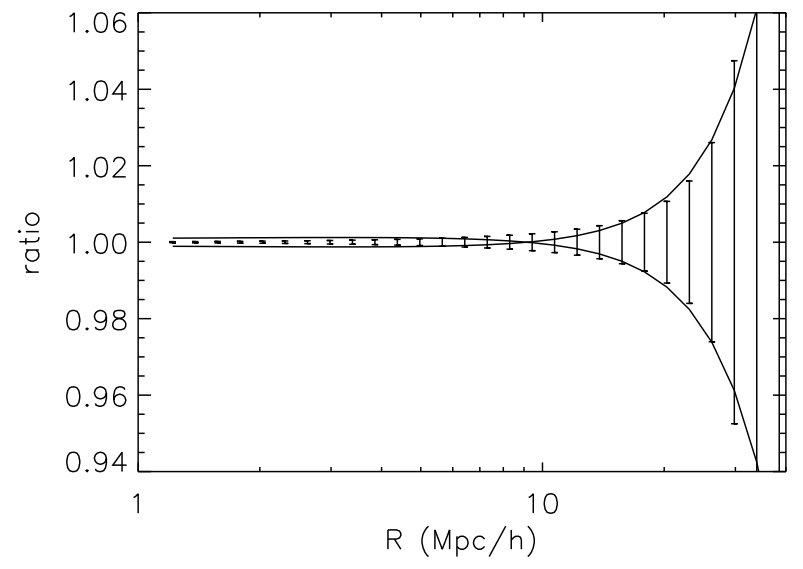

Figure 2: The ratio of the void abundance with non-Gaussianity at the level of $\left|f_{\mathrm{nl}}\right|=30$ to the void abundance with Gaussian initial conditions as a function of the void size $R$. The curves are evaluated for a central redshift $z=0.8$, and the points are $1 \sigma$ Poisson errors for a survey of width $\Delta z=0.3$ that covers 30,000 square degrees for a fiducial Gaussian case. The curve with a large ratio at large $R$ is for $f_{\mathrm{nl}}<0$, while the curve with the small ratio at large $R$ is for $f_{\mathrm{nl}}>0$.

As discussed above, $\left|\delta_{v}\right|$ is expected to be smaller than $\delta_{c}$ by a factor $2-3$. If voids and halos probe the same scales, then voids should yield constraints on $f_{\mathrm{nl}}$ three times worse. However, voids may probe slightly larger scales than halos, and as $S_{3}$ in these models increases with scale, this compensates for $\left|\delta_{v}\right|$ being smaller than $\delta_{c}$.

\section{Results}

To illustrate the promise held by the void abundance for constraining $f_{\mathrm{nl}}$, we consider several large-volume LSS surveys at high redshift that are now being considered. The curves in figure 2 show the ratio of the void abundance with non-Gaussianity to the abundance with Gaussian initial conditions, as a function of void size $R$, for representative values $f_{\mathrm{nl}}=+30$ and $f_{\mathrm{nl}}=-30$. The curves are evaluated for a redshift $z=0.8$. The curve with greater void abundance at larger $R$ and smaller void abundance at smaller $R$ is for negative $f_{\mathrm{nl}}$ and negative skewness in the density field, while that with smaller (larger) void abundance at larger (smaller) $R$ is for positive $f_{\mathrm{nl}}$ (positive skewness in the density field). The crossover in $R$ between enhancement and suppression of the void abundance reflects the onset of the exponential tail of the PS distribution.

The points with error bars estimate the $1 \sigma$ error bars (coming from Poisson errors in the void abundance) anticipated for a survey with a redshift slice $\Delta z=0.3$ centered at $z=0.8$ that covers 30,000 square degrees of the sky. The curves are evaluated for a critical underdensity $\delta_{v}=-0.7$. As indicated by the figure, signal to noise in the determination of $f_{\mathrm{nl}}$ will come primarily from the lower- $R$ end of the distribution, where the void abundance is larger.

Figure 3 shows the smallest local-model $f_{\mathrm{nl}}$ detectable at the $1 \sigma$ level as a function of the critical underdensity $\delta_{v}$ for several surveys currently under study. The solid upper line is a survey with parameters comparable to those proposed for the BOSS SDSS-3 survey [28] (which we parametrize as a 10,000 square-degree survey complete to redshift $z=0.8$ ); this curve 


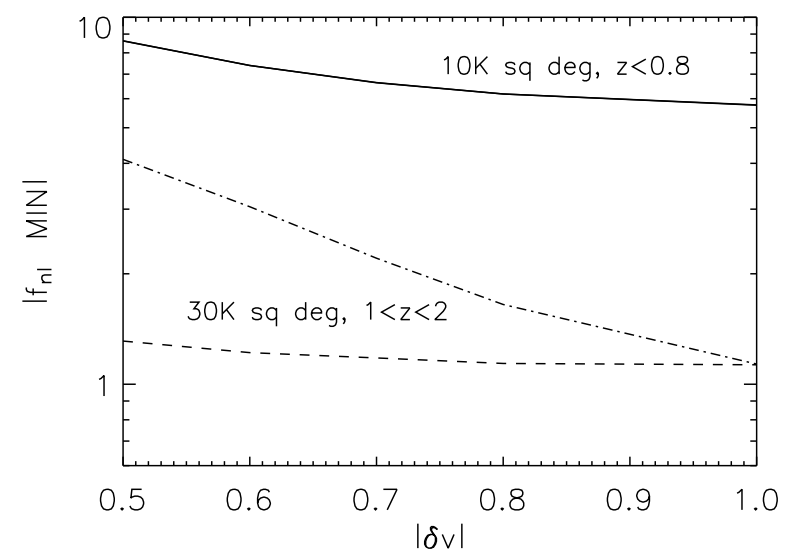

Figure 3: The smallest local-model $f_{\mathrm{nl}}$ detectable at the $1 \sigma$ level, as a function of the critical underdensity $\delta_{v}$, for several surveys currently under study. The solid upper line is for something like the BOSS SDSS-3 survey using the void-size distribution over the range $2 h^{-1} \mathrm{Mpc}<R<60 h^{-1} \mathrm{Mpc}$. The lower two (dashed) curves are for an ADEPT-like survey. The upper dashed curve uses only the void distribution with $R>8 h^{-1} \mathrm{Mpc}$; the lower dashed curve uses $2 h^{-1} \mathrm{Mpc}>R<60 h^{-1} \mathrm{Mpc}$.

is evaluated using the void-size distribution over the range $2 h^{-1} \mathrm{Mpc}<R<60 h^{-1} \mathrm{Mpc}$. The lower two (dashed) lines assume parameters of a survey like ADEPT [29] (which we parametrize as a 30,000 square-degree survey over the redshift range $1<z<2$. The upper dashed curve uses only the void distribution with $R>8 h^{-1} \mathrm{Mpc}$; the lower dashed curve uses $2 h^{-1} \mathrm{Mpc}<R<60 h^{-1} \mathrm{Mpc}$.

Since the void abundance depends on non-Gaussianity only through the dependence on $S_{3, R}$, we can use the curves shown in figure 1 to compute constraints for the equilateral model, both with and without scale dependence. As expected, since the equilateral model (with no scale dependence) predicts a value of $S_{3, R}$ that is about 3 times smaller than in the local model, the smallest the values of $f_{\mathrm{nl}}^{\mathrm{eq}}$ accessible with the void abundance is larger by roughly a factor of 3 than in the local model. Likewise, figure 3 shows that $f_{\text {nl }}$ for the equilateral model with $\kappa=-0.3$ is comparable to that in the local model; the constraints to $f_{\mathrm{nl}}^{\mathrm{eq}}$ for $\kappa=-0.3$ are similar to those in the local model. This is particularly interesting as the large-scale bias effect due to equilateral non-gaussianity is orders of magnitude smaller than the effect for the local case [27]. Thus the combination of the two measurements will help discriminate among different type of non-gaussian initial conditions.

As figure 3 shows, the results do not depend very sensitively on the precise value of $\delta_{v}$. The value that we choose for $\delta_{v}$ depends on the precise definition of a void. Here we have used as a canonical value $\delta_{v}=-0.7$ for the critical linear-theory underdensity for a void. At this value of $\delta_{v}$, the physical void is underdense by a factor of 2 (see, e.g., figure 1 in ref. [23]), which we believe to be a conservative definition of a void. If voids are defined in the survey to be regions that are even emptier, then the relevant magnitude $\left|\delta_{v}\right|$ will be even larger, and according to figure 3 , the smallest detectable $\left|f_{\text {nl }}\right|$ will be even a bit smaller. 


\section{Discussion}

The bottom line of our analysis is that the abundance of voids in LSS surveys that are currently being considered can probe values of the non-Gaussianity parameter in the local model as small as $f_{\mathrm{nl}} \sim 10$ and in the equilateral model (with no scale dependence) as small $f_{\mathrm{nl}}^{\mathrm{eq}} \sim 30$. This probe of non-Gaussianity may be competitive with those coming from the CMB, LSS, and cluster abundances [10, 15, 20]. They will complement CMB constraints by (a) providing a different avenue with different systematic effects, and (b) probing nonGaussianity primarily over distance scales $2-60 \mathrm{Mpc}$, scales generally smaller than those that will be probed by the CMB. The fact that voids constraints on the different types of non gaussianity are comparable is particularly interesting. In fact the large-scale bias effect due to equilateral non-gaussianity is orders of magnitude smaller than the effect for the local case. Thus the combination of the two measurements will help discriminate among different type of non-gaussian initial conditions.

Finally, we note that we have here done no more than estimate the smallest $f_{\text {nl }}$ detectable with the void abundance. To do so, we have taken a simple Press-Schechter approach to estimate the fractional change in the void abundance. While this approach should provide reasonable rough estimates to the fractional change in the abundance, it will not reliably provide the abundances themselves. Before the void-abundance probe of $f_{\mathrm{nl}}$ can be implemented, we will therefore require significantly more sophisticated calculations, which will probably require numerical simulations, to accurately model not only the void growth, but also the systematic effects that will arise in any realistic void-identification algorithm. We hope that our results motivates this type of future work.

\section{Acknowledgments}

MK thanks Andrew Benson for useful suggestions and the Aspen Center for Physics for hospitality. This work was supported at Caltech by DoE DE-FG03-92-ER40701 and the Gordon and Betty Moore Foundation. LV thanks M. LoVerde for providing the curves of figure 3(a,b) of ref. [20] in table form. LV is supported by FP7-PEOPLE-2007-4-3 IRG $\mathrm{n}$ 202182 and CSIC I3 grant 200750I034. RJ is supported by grants from the Spanish Science Ministry and The European Union (FP7).

\section{References}

[1] Boomerang collaboration, P. de Bernardis et al., A flat universe from high-resolution maps of the cosmic microwave background radiation, Nature 404 (2000) 955 [astro-ph/0004404] [SPIRES];

A.D. Miller et al., A measurement of the angular power spectrum of the CMB from $l=100$ to 400, Astrophys. J. 524 (1999) L1 [astro-ph/9906421] [SPIRES];

S. Hanany et al., MAXIMA-1: a measurement of the cosmic microwave background anisotropy on angular scales of 10 arcminutes to 5 degrees,

Astrophys. J. 545 (2000) L5 [astro-ph/0005123] [SPIRES];

N.W. Halverson et al., DASI first results: a measurement of the cosmic microwave background angular power spectrum, Astrophys. J. 568 (2002) 38 [astro-ph/0104489] [SPIRES]; 
B.S. Mason et al., The anisotropy of the microwave background to $l=3500$ : deep field observations with the cosmic background imager, Astrophys. J. 591 (2003) 540 [astro-ph/0205384] [SPIRES];

The Archeops collaboration, A. Benoit et al., Cosmological constraints from Archeops, Astron. Astrophys. 399 (2003) L25 [astro-ph/0210306] [SPIRES];

J.H. Goldstein et al., Estimates of cosmological parameters using the CMB angular power spectrum of ACBAR, Astrophys. J. 599 (2003) 773 [astro-ph/0212517] [SPIRES];

WMAP collaboration, D.N. Spergel et al., First year Wilkinson Microwave Anisotropy Probe (WMAP) observations: determination of cosmological parameters, Astrophys. J. Suppl. 148 (2003) 175 [astro-ph/0302209] [SPIRES];

C.L. Reichardt et al., High resolution CMB power spectrum from the complete ACBAR data set, arXiv:0801.1491 [SPIRES];

WMAP collaboration, D.N. Spergel et al., Wilkinson Microwave Anisotropy Probe (WMAP) three year results: implications for cosmology, Astrophys. J. Suppl. 170 (2007) 377 [astro-ph/0603449] [SPIRES].

[2] N. Bartolo, E. Komatsu, S. Matarrese and A. Riotto, Non-Gaussianity from inflation: theory and observations, Phys. Rept. 402 (2004) 103 [astro-ph/0406398] [SPIRES].

[3] A.H. Guth and S.Y. Pi, Fluctuations in the new inflationary universe, Phys. Rev. Lett. 49 (1982) 1110 [SPIRES];

A.A. Starobinsky, Dynamics of phase transition in the new inflationary universe scenario and generation of perturbations, Phys. Lett. B 117 (1982) 175 [SPIRES]; J.M. Bardeen, P.J. Steinhardt and M.S. Turner, Spontaneous creation of almost scale free density perturbations in an inflationary universe, Phys. Rev. D 28 (1983) 679 [SPIRES].

[4] T. Falk, R. Rangarajan and M. Srednicki, The angular dependence of the three point correlation function of the cosmic microwave background radiation as predicted by inflationary cosmologies, Astrophys. J. 403 (1993) L1 [astro-ph/9208001] [SPIRES];

A. Gangui, F. Lucchin, S. Matarrese and S. Mollerach, The three point correlation function of the cosmic microwave background in inflationary models, Astrophys. J. 430 (1994) 447 [astro-ph/9312033] [SPIRES];

A. Gangui, NonGaussian effects in the cosmic microwave background from inflation, Phys. Rev. D 50 (1994) 3684 [astro-ph/9406014] [SPIRES];

L.-M. Wang and M. Kamionkowski, The cosmic microwave background bispectrum and inflation, Phys. Rev. D 61 (2000) 063504 [astro-ph/9907431] [SPIRES].

[5] D. Babich, P. Creminelli and M. Zaldarriaga, The shape of non-Gaussianities, JCAP 08 (2004) 009 [astro-ph/0405356] [SPIRES];

P. Creminelli, A. Nicolis, L. Senatore, M. Tegmark and M. Zaldarriaga, Limits on non-Gaussianities from WMAP data, JCAP 05 (2006) 004 [astro-ph/0509029] [SPIRES];

P. Creminelli, L. Senatore, M. Zaldarriaga and M. Tegmark, Limits on $f_{N L}$ parameters from WMAP 3 yr data, JCAP 03 (2007) 005 [astro-ph/0610600] [SPIRES].

[6] T.J. Allen, B. Grinstein and M.B. Wise, Nongaussian density perturbations in inflationary cosmologies, Phys. Lett. B 197 (1987) 66 [SPIRES]; 
L.A. Kofman and D.Y. Pogosian, Nonflat perturbations in inflationary cosmology, Phys. Lett. B 214 (1988) 508 [SPIRES];

D.S. Salopek, J.R. Bond and J.M. Bardeen, Designing density fluctuation spectra in inflation, Phys. Rev. D 40 (1989) 1753 [SPIRES];

A.D. Linde and V.F. Mukhanov, NonGaussian isocurvature perturbations from inflation, Phys. Rev. D 56 (1997) 535 [astro-ph/9610219] [SPIRES];

P.J.E. Peebles, An isocurvature CDM cosmogony. I. A worked example of evolution through inflation, astro-ph/9805194 [SPIRES]; An isocurvature CDM cosmogony. II. Observational tests, astro-ph/9805212 [SPIRES].

[7] S. Mollerach, Isocurvature baryon perturbations and inflation, Phys. Rev. D 42 (1990) 313 [SPIRES];

A.D. Linde and V.F. Mukhanov, NonGaussian isocurvature perturbations from inflation, Phys. Rev. D 56 (1997) 535 [astro-ph/9610219] [SPIRES];

D.H. Lyth and D. Wands, Generating the curvature perturbation without an inflaton, Phys. Lett. B 524 (2002) 5 [hep-ph/0110002] [SPIRES];

T. Moroi and T. Takahashi, Effects of cosmological moduli fields on cosmic microwave background, Phys. Lett. B 522 (2001) 215 [Erratum ibid. B 539 (2002) 303] [hep-ph/0110096] [SPIRES];

D.H. Lyth, C. Ungarelli and D. Wands, The primordial density perturbation in the curvaton scenario, Phys. Rev. D 67 (2003) 023503 [astro-ph/0208055] [SPIRES]; K. Ichikawa, T. Suyama, T. Takahashi and M. Yamaguchi, Non-gaussianity, spectral index and tensor modes in mixed inflaton and curvaton models, Phys. Rev. D 78 (2008) 023513 [arXiv:0802.4138] [SPIRES];

A.L. Erickcek, M. Kamionkowski and S.M. Carroll, A hemispherical power asymmetry from inflation, arXiv:0806.0377 [SPIRES].

[8] G.R. Dvali and S.H.H. Tye, Brane inflation, Phys. Lett. B 450 (1999) 72 [hep-ph/9812483] [SPIRES];

P. Creminelli, On non-Gaussianities in single-field inflation, JCAP 10 (2003) 003 [astro-ph/0306122] [SPIRES];

M. Alishahiha, E. Silverstein and D. Tong, DBI in the sky,

Phys. Rev. D 70 (2004) 123505 [hep-th/0404084] [SPIRES].

[9] X.-C. Luo, The angular bispectrum of the cosmic microwave background, Astrophys. J. 427 (1994) L71 [astro-ph/9312004] [SPIRES].

[10] L. Verde, L.-M. Wang, A. Heavens and M. Kamionkowski, Large-scale structure, the cosmic microwave background and primordial non-Gaussianity, Mon. Not. Roy. Astron. Soc. 313 (2000) L141 [astro-ph/9906301] [SPIRES].

[11] E. Komatsu and D.N. Spergel, Acoustic signatures in the primary microwave background bispectrum, Phys. Rev. D 63 (2001) 063002 [astro-ph/0005036] [SPIRES].

[12] P. Coles, L. Moscardini, F. Lucchin, S. Matarrese and A. Messina, Skewness as a test of nonGaussian primordial density fluctuations, Mon. Not. Roy. Astron. Soc. 264 (1993) 749 [astro-ph/9302015] [SPIRES];

X.-C. Luo and D.N. Schramm, Kurtosis, skewness and nonGaussian cosmological density perturbations, Astrophys. J. 408 (1993) 33 [SPIRES]; 
E.L. Lokas, R. Juszkiewicz, D.H. Weinberg and F.R. Bouchet, Kurtosis of large scale cosmic fields, Mon. Not. Roy. Astron. Soc. 274 (1995) 730 [astro-ph/9407095] [SPIRES];

M.J. Chodorowski and F.R. Bouchet, Kurtosis in large-scale structure as a constraint on non-gaussian initial conditions, Mon. Not. Roy. Astron. Soc. 279 (1996) 557 [astro-ph/9507038] [SPIRES];

A.J. Stirling and J.A. Peacock, Power correlations in cosmology: limits on primordial non-Gaussian density fields, astro-ph/9608101 [SPIRES];

R. Durrer, R. Juszkiewicz, M. Kunz and J.-P. Uzan, Skewness as a probe of nonGaussian initial conditions, Phys. Rev. D 62 (2000) 021301 [astro-ph/0005087] [SPIRES];

L. Verde and A.F. Heavens, On the trispectrum as a gaussian test for cosmology, Astrophys. J. 553 (2001) 14 [astro-ph/0101143] [SPIRES].

[13] W.A. Chiu, J.P. Ostriker and M.A. Strauss, Using cluster abundances and peculiar velocities to test the gaussianity of the cosmological density field,

Astrophys. J. 494 (1998) 479 [astro-ph/9708250] [SPIRES];

J. Robinson, E. Gawiser and J. Silk, Constraining primordial non-gaussianity with the abundance of high redshift clusters, Astrophys. J. 532 (2000) 1 [astro-ph/9906156] [SPIRES];

J.A. Willick, Constraints on primordial nongaussiantiy from the high-redshift cluster MS1054-03, astro-ph/9904367 [SPIRES];

L. Verde, M. Kamionkowski, J.J. Mohr and A.J. Benson, On galaxy-cluster sizes and temperatures, Mon. Not. Roy. Astron. Soc. 321 (2001) L7 [astro-ph/0007426] [SPIRES];

N.N. Weinberg and M. Kamionkowski, Constraining dark energy from the abundance of weak gravitational lenses, Mon. Not. Roy. Astron. Soc. 341 (2003) 251 [astro-ph/0210134] [SPIRES].

[14] S. Matarrese, L. Verde and R. Jimenez, The abundance of high-redshift objects as a probe of non-Gaussian initial conditions, Astrophys. J. $\mathbf{5 4 1}$ (2000) 10 [astro-ph/0001366] [SPIRES].

[15] L. Verde, R. Jimenez, M. Kamionkowski and S. Matarrese, Tests for primordial non-Gaussianity, Mon. Not. Roy. Astron. Soc. 325 (2001) 412 [astro-ph/0011180] [SPIRES].

[16] N. Dalal, O. Dore, D. Huterer and A. Shirokov, The imprints of primordial non-Gaussianities on large-scale structure: scale dependent bias and abundance of virialized objects, Phys. Rev. D 77 (2008) 123514 [arXiv:0710.4560] [SPIRES].

[17] S. Matarrese and L. Verde, The effect of primordial non-Gaussianity on halo bias, Astrophys. J. Lett. 677 (2008) L77 [arXiv:0801.4826] [SPIRES].

[18] A. Slosar, C. Hirata, U. Seljak, S. Ho and N. Padmanabhan, Constraints on local primordial non-Gaussianity from large scale structure, JCAP 08 (2008) 031 [arXiv:0805.3580] [SPIRES].

[19] C. Carbone, L. Verde and S. Matarrese, Non-Gaussian halo bias and future galaxy surveys, arXiv:0806.1950 [SPIRES]. 
[20] M. LoVerde, A. Miller, S. Shandera and L. Verde, Effects of scale-dependent non-Gaussianity on cosmological structures, JCAP 04 (2008) 014 [arXiv:0711.4126] [SPIRES].

[21] M. Grossi, E. Branchini, K. Dolag, S. Matarrese and L. Moscardin, The mass density field in simulated non-Gaussian scenarios, arXiv:0805.0276 [SPIRES].

[22] R.K. Sheth and R. van de Weygaert, A hierarchy of voids: much ado about nothing, Mon. Not. Roy. Astron. Soc. 350 (2004) 517 [astro-ph/0311260] [SPIRES].

[23] S. Furlanetto and T. Piran, The evidence of absence: galaxy voids in the excursion set formalism, Mon. Not. Roy. Astron. Soc. 366 (2006) 467 [astro-ph/0509148] [SPIRES].

[24] M. Grossi et al., The non-gaussian mass function, in preparation.

[25] G.R. Blumenthal, L. Nicolaci da Costa, D.S. Goldwirth, M. Lecar and T. Piran, The largest possible voids, Astrophys. J. 388 (1992) 234 [SPIRES].

[26] WMAP collaboration, E. Komatsu et al., First year Wilkinson Microwave Anisotropy Probe (WMAP) observations: tests of Gaussianity, Astrophys. J. Suppl. 148 (2003) 119 [astro-ph/0302223] [SPIRES];

A.P.S. Yadav and B.D. Wandelt, Evidence of primordial non-Gaussianity $\left(f_{\mathrm{NL}}\right)$ in the Wilkinson Microwave Anisotropy Probe 3-year data at 2.8 $\sigma$,

Phys. Rev. Lett. 100 (2008) 181301 [arXiv:0712.1148] [SPIRES];

WMAP collaboration, E. Komatsu et al., Five-year Wilkinson Microwave Anisotropy Probe (WMAP1) observations: cosmological interpretation, arXiv:0803.0547 [SPIRES].

[27] A. Taruya, K. Koyama and T. Matsubara, Signature of primordial non-Gaussianity on matter power spectrum, arXiv:0808.4085 [SPIRES];

L. Verde et al., Non-gaussianity and the matter power spectrum, in preparation.

[28] BOSS: dark energy and the geometry of space, http://www.sdss3.org/cosmology.php.

[29] ADEPT, the Advanced Dark Energy Physics Telescope, http://universe.nasa.gov/program/probes/adept.html. 